\title{
Modular and Integrated Sensor Network of Intelligent Radiation Monitor Systems for Radiological and Nuclear Threat Response
}

\author{
M. Corbo*, M. Morichi, E. Fanchini, G. Mini, A. Pepperosa, G. Mangiagalli \\ * CAEN SyS s.r.l., Viareggio, Italy \\ m.corbo@caensys.com
}

\begin{abstract}
Increased sensitivity to nuclear safety and security issues has prompted public entities and private institutions to maximize their capability to rapidly assess risks and intervene in the case of accident or threat. Quick intervention and response are achieved through nuclear measurements via airborne, land, and underwater systems. A network of cohesive, well-integrated and easy deployable radiation monitoring systems combined with realtime analysis of data is essential to facilitate and enhance the decision-making process during these most critical moments enhancing the quality of the management plan.

The presented radiation monitoring systems can be integrated in several form factors which depend mainly on operational needs and internal battery for autonomous operation. Compact ARM based computers is embedded, which can store large amount of data in their non-volatile memory, run automatic data analysis and trigger alarms in case of exceeding radiation levels. All the systems can communicate with redundant interfaces in failover configuration and upload the acquired environmental information in a central database. The same monitoring systems can alert the emergency response personnel on the field as well, through wireless connection to common tablets or cellphone or SMS, guaranteeing a prompt response in case an illicit transportation of radiological or nuclear material is detected.
\end{abstract}

Keywors-Radiological Monitoring, Environmental Monitoring, Spectrometer, Nuclear Security, Autonomous Stations.

\section{I.INTRODUCTION}

AUTORITIES have the necessity to monitor the radiological situation and implement actions to minimize radiological effects on the population. One common issue, in case of an alarm, is that the available measurement systems do not communicate directly to the operator causing important delays in the decision-making process necessary to define the assessment and mitigation plans. Furthermore, some operations require the use of several measurement systems and in case these systems were not designed to be integrated into a network, results cannot be analysed together in real time, requiring a time consuming offline post-processing. From here the necessity to have a real time network of integrable systems for a precise and immediate evaluation of the situation.

Current embedded commercial electronics, as compact ARM based computers, allow the integration of intelligent radiation monitoring systems meeting the needs of integrated solutions for radiological and nuclear security. The building blocks of the radiation monitoring systems can be easily programmed for different operations remotely or locally. The working condition can be monitored real time and in case of malfunctioning the optimization of the data acquisition can be performed remotely as well. Access credentials and secured data transmission avoid that unauthorized people could read or delete the acquired data and avoid that operators on field could accidentally modify hardware or software configuration.

Web based software instruments ease, at the same time, the design of user-friendly graphical interfaces and guarantees a good level of data security as mentioned. A common software platform can manage the information from different radiation detectors giving the possibility to arrange the network of deployed sensors in a modular way.

\section{DESCRIPTION OF HARDWARE}

The intelligent radiation monitoring systems are composed by some elements that are reported here below.

- The low power consumption and compact microprocessor

- The spectrometer composed by inorganic scintillators for gamma radiation identification; thanks to innovative scintillation detectors it is possible to identify neutron and gamma radiation at the same time as well

- The gamma and neutron counters as Geiger-Muller or $3 \mathrm{He}$ tubes.

The intelligent radiation monitoring systems are configured in different form factors to match the needs of all possible operation scenarios: compact and discrete to be carried in a backpack; lightweight and provided with wireless data transmission to be transported on unmanned aerial drones; rugged designed with high efficiency detectors to be transported on vehicles; tolerant to a wide temperature range and harsh weather conditions to be installed as autonomous stations in open field; waterproof to monitor the contamination in water or in the sediments of rivers, lakes, seas and oceans.

\section{A. Microprocessor}

The microprocessor is based on an ARM which stores the data in an internal non-volatile memory of more than $8 \mathrm{~GB}$. Like other commercially available microprocessors it is provided with multiple communication interfaces, like Ethernet, USB and serial ports, and multiple inputs and outputs, which can be 
programmed for connecting peripherals and connect radiation counters. The operator can select the preferred communication interface and backup interfaces, which guarantee the communication thanks with failover capability.

Commonly available rain sensors, meteorological stations, GPS receivers, antitampering sensors can be connected to the microprocessor to provide additional functionalities and improving the ability to respond to a wide range of operational activities.

The microprocessor runs a web interface which gives the user the possibility to easily configure the data acquisition and the isotopic analysis.

\section{B. Spectrometer}

The gamma spectrometer is composed by an inorganic scintillation crystal which can be either $\mathrm{NaI}(\mathrm{Tl}), \mathrm{CeBr}_{3}$ or $\mathrm{LaBr}_{3}(\mathrm{Ce}) . \mathrm{NaI}(\mathrm{Tl})$ is preferable its high light yield and moderate cost, $\mathrm{LaBr}_{3}(\mathrm{Ce})$ for its excellent energy resolution and $\mathrm{CeBr} 3$ for its good energy resolution and low internal radioactivity.

The innovative NaIL TM [1] detector can be integrated in the radiation monitoring systems to obtain high efficiency thermal neutron detection combined with gamma ray spectrometry. This allows the system to detect the presence of both gamma ray emitters and identify special nuclear material. E.g. Fig. 1 shows the data acquired in presence of $238 \mathrm{Pu}$ source with a neutron yield of $6.5 \mathrm{n} / \mathrm{s}$ with $5 \mathrm{~cm}$ of Polyethylene.

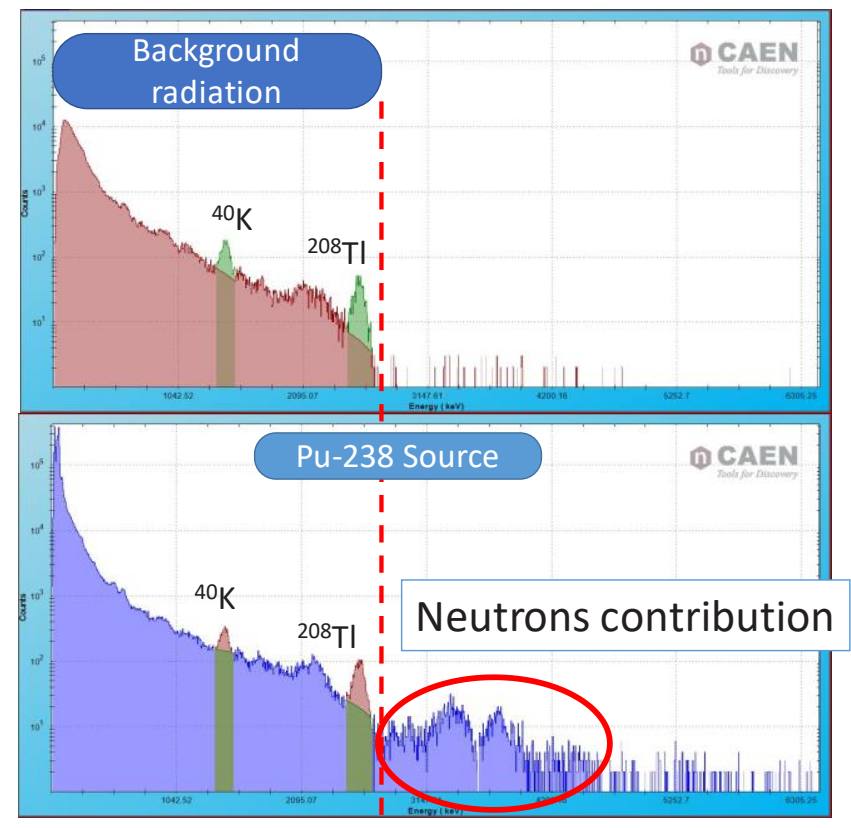

Fig. 1: Spectra acquired with a NAIL detector 2"x2" at $0.5 \%$ Lithium doping. The spectrum above represents the background, while the spectrum below shows the data acquired in presence of $238 \mathrm{Pu}$ source with a neutron yield of $6.5 \mathrm{n} / \mathrm{s}$ with $5 \mathrm{~cm}$ of Polyethylene

Signals from scintillation detector is conditioned by a preamplifier, the pulse is digitized by a 12-bit $62.5 \mathrm{MHz}$ flash Analog to Digital Converter and the corresponding data flow is analysed by a Field Programmable Gate Array performing a Digital Pulse Processing (DPP) [2]. The DPP can be optimized for different detector types thanks to the programmable parameters of the digital discriminator and the digital shaping for pulse height analysis.

The spectroscopy detector is configured to collect gamma interactions in the energy range from $50 \mathrm{keV}$ to $3 \mathrm{MeV}$, even though alternative energy ranges can be easily configured on request. The spectrometer runs isotope identification and isotope related dose rate evaluation automatically while acquiring the data. The real-time data processing and the defined isotope-based alarm allow system to autonomous detect the presence of artificial nuclides in a short time window and more effectively compared to traditional dose rate meters. The user can select the isotopes to be identified from the library and adjust the thresholds of the isotope related alarms. It provides statistically accurate dose rate measurements in the wide range from $1 \mathrm{nSv} / \mathrm{h}$ to $100 \mu \mathrm{Sv} / \mathrm{h}$.

The spectrum stabilization is obtained with the identification of natural occurring radionuclides as the ${ }^{40} \mathrm{~K}$ and rescaling the calibration of the spectrum to maintain the centroid of ${ }^{40} \mathrm{~K}$ peak at the right position in the spectrum.

\section{Gamma or Neutron Counters}

Geiger-Muller or neutron counters can be connected to the microprocessor inputs and outputs. Compensated GeigerMuller could effectively measure the Ambient Dose Equivalent, while ${ }^{3} \mathrm{He}$ or boron lined tubes could effectively detect neutrons from special nuclear material.

\section{SOFTWARE}

One of the main advantages of the commonly used web application is the multiplatform compatibility, which means that the intelligent radiation monitoring systems can be monitored from different types of devices and their information can be received from tablets or smartphones. This gives the security or emergency response personnel the possibility to promptly detect threats on field.

The web-based application is a webserver which may run either in the deployed monitoring systems or in a central monitoring facility with gathers together the information from a network of systems. A database is present both in the monitoring system and in the central monitoring facility as well, enabling the possibility to select and download data reports depending on user defined criteria. This capability is fundamental for operators to promptly and effectively diagnose an emergency scenario or to easily select the data of monitoring systems in a comprehensive nationwide network.

\section{A. Web Application of the Intelligent Radiation Monitoring Systems}

The intelligent radiation monitoring systems are provided with a web server application running in the internal central unit processor. The radiological information is stored in an internal database and in the wide non-volatile memory, allowing autonomous data taking even in case of sudden connection lost. Spectra, acquisition reports, data history can be displayed and downloaded through a common web browser. Users can monitor the data or modify the acquisition settings depending on the level of access credentials: user, technician or 
administrator. E.g. Fig. 2 represents the administrator page for setting the operating parameters of the spectrometer.

Spectral analysis and statistical data treatment are performed, and extended isotope library is used for identification routines to address the robust spectrum stabilization algorithms are implemented. The user can perform long term quality checks and even recalibrate the system in case of need.

The station can signal radiological alarms or malfunctions automatically to the central monitoring facility or directly to operators through phone messages or emails. Different levels of alarm can be configured on the dosimeter measurement, on the neutron counting, on the spectrometer count rate and on the dose rate associated to identified peaks in the spectrum.

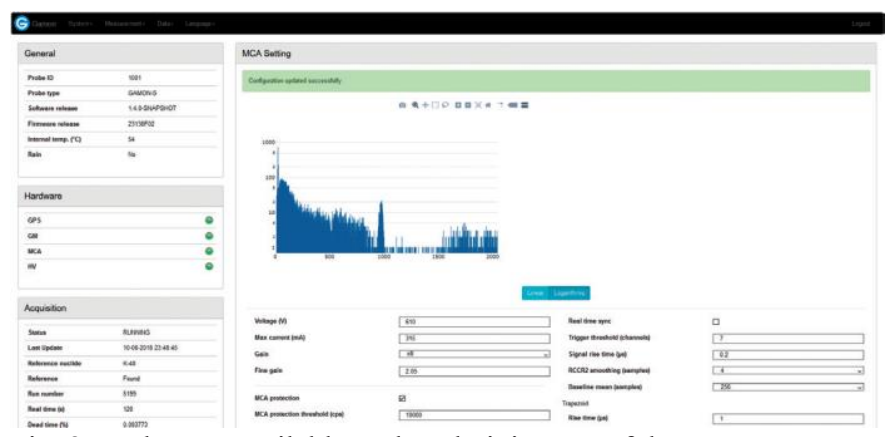

Fig. 2: Web page available to the administrator of the system, to set the operational parameter of the spectrometer

\section{B. Web application for the Network of Systems}

The web application for the network is an intuitive and easy handling tool for the operator to supervise all intelligent radiation monitoring systems deployed in the field by a userfriendly web interface. The intuitive graphical interface allows the user to fully control the situation guaranteeing fast and reliable decision making for prompt emergency response.

The software visualizes all the network of systems on a map showing the status of all deployed systems and the radiological situation, alerting the operator in case of alarm. The dosimetric heat map is obtained using data fusion algorithms using real time measurements for all georeferenced systems.

The network software is the perfect tool for wide area coverage applications or the monitoring of sensitive locations or events to have a safer and clearer monitoring overview.

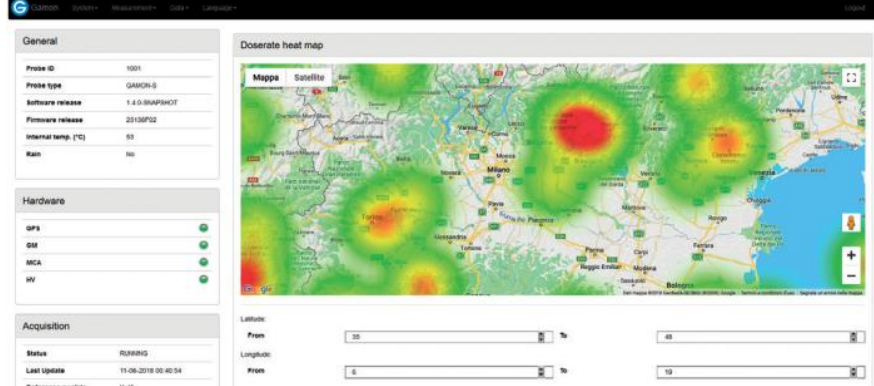

Fig. 3: Web page to visualize the count rate, dose rate or isotope presence in the form of a heat map

\section{GAMON-S CHARACTERIZATION}

The GAMON-S System has been characterized under fast and extreme temperature changes and the radiological measurements have been verified in laboratory and Monte Carlo method. In this section we report the most relevant features and performance that have been measured.

\section{A. Environmental Chamber Test}

The GAMON-S System has been tested in environmental chamber to assess the temperature stabilization capability of the software algorithms. The environmental chamber has been configured to obtain a stress test with a wide range temperature cycle, with extreme temperatures -40 and $+60{ }^{\circ} \mathrm{C}$, to be performed in 24 hours cycle.

A radioactive source of $\mathrm{Cs}-137$ has been used to create a reference peak for the spectrum stabilization technique. Internal temperature of the crystal has been monitored and the position of the stabilization peak has been correlated to the measured temperature. Fig. 4 represents the trend of the reference peak position over time, together with the monitored temperature. On the overall the peak stabilization algorithm guarantees the stability over a total range of $\pm 0.5 \%$ respect to the central reference peak position. This performance allows the algorithm of radioisotope identification to not fail in case of quick changes of external temperature.

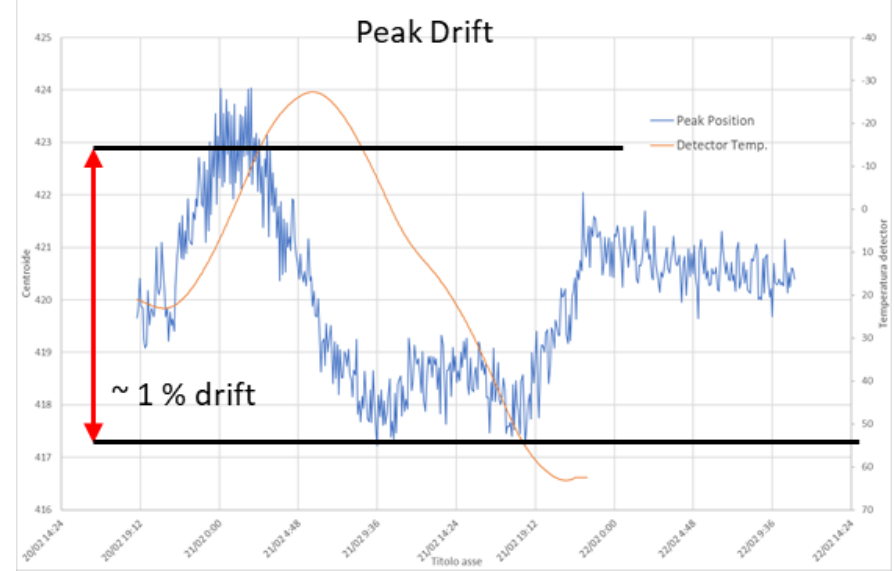

Fig. 4: In blue the reference peak position over the $24 \mathrm{~h}$ temperature cycle. In orange the measured temperature of the $\mathrm{CeBr} 3$ scintillator crystal (ranging from -30 to $+65^{\circ} \mathrm{C}$ )

\section{B. Spectrometer Response to Ambient Equivalent Dose $H^{*}(10)$}

The GAMON-S has been characterized for its response to the Ambient Equivalent Dose $\mathrm{H}^{*}(10)$ through laboratory measurements and a comparative study with a simulation performed with MCNP 6.1.1.

The simulation at MCNP 6.1.1 has been done using the geometry of a 1.5 "x1.5" $\mathrm{CeBr} 3$ crystal together with the aluminum case, reflective material and mu-metal cover in the proximity of the crystal itself. The scintillator crystal has been inserted inside sections of pipes having the thickness and material type of the GAMON-S cases. The flux of radiation has been generated perpendicular to the $\mathrm{CeBr} 3$ crystal axis with uniform distribution of parallel gamma rays ranging from 30 $\mathrm{keV}$ to $3 \mathrm{MeV}$. This simulation has been used in conjunction with the data reported in the ICRU Report N.57 to compute the 
conversion factors between the number of counts in the gamma ray photopeak and the associated Ambient Equivalent Dose $\mathrm{H}^{*}(10)$.

The result of the simulation has been compared with experimental measurements performed with ${ }^{241} \mathrm{Am}$ and ${ }^{137} \mathrm{Cs}$ point sources at 20 and $30 \mathrm{~cm}$ distance from the center of the detector axe perpendicular to its axis. The result of the comparison is reported in the table below, which demonstrate a good agreement at the energy of the $661.7 \mathrm{keV}$ gamma line, better than $5 \%$, and sufficient agreement at the energy of 59.5 $\mathrm{keV}$, with a disagreement of the order of $20 \%$.

\begin{tabular}{l|lllll}
$\begin{array}{l}\text { Nuclide } \\
\text { (Act.) }\end{array}$ & $\begin{array}{l}\text { Energy } \\
{[\mathrm{keV}]}\end{array}$ & $\begin{array}{l}\text { D. } \\
\text { [cm] }\end{array}$ & $\begin{array}{l}\text { Measured } \\
{[\mathrm{nSv} / \mathrm{h} / \mathrm{cps}]}\end{array}$ & $\begin{array}{l}\text { Simulated } \\
{[\mathrm{nSv} / \mathrm{h}} \\
\mathrm{cps}]\end{array}$ & Discrepancy \\
\hline $\begin{array}{l}{ }^{241} \mathrm{Am} \\
(\mathbf{3}, \mathbf{4 3} \mathbf{~ k B q})\end{array}$ & 59,5 & 20 & $0.2387 \pm 2.0 \%$ & 0.2061 & $13.7 \%$ \\
& & 30 & $0.2670 \pm 3.1 \%$ & 0.2061 & $22.8 \%$ \\
$\begin{array}{l}{ }^{137} \mathrm{Cs}(\mathbf{2 3 . 0} \\
\text { kBq) }\end{array}$ & 661,7 & 20 & $3.9090 \pm 1.1 \%$ & 3.7927 & $3.0 \%$ \\
& & 30 & $3.9881 \pm 1.7 \%$ & 3.7927 & $4.9 \%$
\end{tabular}

\section{Spectrometer Linearity}

The GAMON-S has been tested at the secondary metrology laboratory at Polytechnical University of Milan to monitor the system linearity as function of the incident instantaneous radiation equivalent dose $H^{*}(10)$. The test has been performed at the hot chamber at Milan with the use of a ${ }^{137} \mathrm{Cs}$ source. In the table below the results of the measurement.

\begin{tabular}{l|llll} 
Nominal $\mathbf{H}^{*}(\mathbf{1 0})$ & $\mathbf{H}^{*}(\mathbf{1 0})$ measured & Discrep. & $\begin{array}{l}\text { Peak } \\
\text { position } \\
{[\mathrm{keV}]}\end{array}$ & $\begin{array}{l}\text { Peak } \\
\text { Net } \\
\text { Area } \\
{[\mathrm{cps}]}\end{array}$ \\
\hline $\mathbf{1 3 . 9 0} \mu \mathrm{Sv} / \mathrm{h} \pm \mathbf{5 . 1 0} \%$ & $13.90 \mu \mathrm{Sv} / \mathrm{h} \pm 0.20 \%$ & $+0.0 \%$ & 656.9 & 2604.9 \\
$\mathbf{2 4 . 7 0} \mu \mathrm{Sv} / \mathrm{h} \pm \mathbf{5 . 4 2 \%}$ & $24.82 \mu \mathrm{Sv} / \mathrm{h} \pm 0.17 \%$ & $+0.5 \%$ & 659.1 & 4652.2 \\
$\mathbf{1 0 3 . 0 0} \mu \mathrm{Sv} / \mathrm{h} \pm \mathbf{6 . 0 1} \%$ & $104.72 \mu \mathrm{Sv} / \mathrm{h} \pm 0.11 \%$ & $+1.7 \%$ & 664.5 & 19624.9 \\
$\mathbf{1 4 8 . 0 0} \mu \mathrm{Sv} / \mathrm{h} \pm \mathbf{6 . 4 2} \%$ & $147.88 \mu \mathrm{Sv} / \mathrm{h} \pm 0.11 \%$ & $-0.1 \%$ & 667.9 & 27712.3
\end{tabular}

\section{SYSTEMS OF THE PLATFORM}

The radiation monitoring systems can be integrated in several form factors which depend mainly on type of deployment. From this perspective, the radiation monitoring systems can be compact to be carried in a backpack or handheld, lightweight to be transported on unmanned aerial drones, rugged designed to be transported on vehicles, tolerant to a wide temperature range and water immersion to be installed in open field.

Here below the description the various version of the intelligent radiation monitoring systems is reported.

\section{A. Gamma Radiation Spectroscopy System for Real-Time Radiation Monitor}

The gamma radiation spectroscopy system represented in Fig. 5 is designed for early environmental warning and emergency response. It runs automatic real-time analysis of surrounding gamma emitting isotopes for detecting radiological threats.

On-field installation allows the system to easily monitor wide surrounding area, detect small deviation from natural background thanks to the high detector efficiency, to provide detailed reports on the radioisotopes found. It has been designed and assembled with a robust case designed to guarantee IP68 and for operating outdoor in extreme weather conditions from 40 to $+60{ }^{\circ} \mathrm{C}$.

To be operated as a station it mounts batteries, optional solar panels and is provided with wired and wireless communication interfaces: USB 2.0, Ethernet, WiFi and 3G/4G LTE.

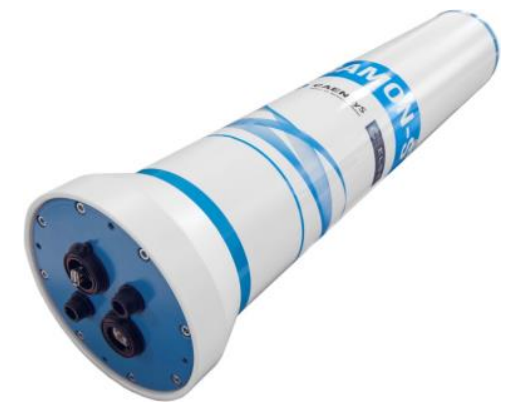

Fig. 5: Picture of the gamma radiation spectroscopy system for realtime radiation monitoring

\section{B. Gamma Dose Rate Monitoring System for Real-Time Radiation Monitoring}

The system is a versatile gamma dose rate monitoring system designed for early environmental warning and emergency response with wide measurement range from $10 \mathrm{nSv} / \mathrm{h}$ to 10 $\mathrm{Sv} / \mathrm{h}$ with subtraction of intrinsic background. The gamma dose rate monitoring system integrates a low power consumption CPU, to guarantee autonomous operations, including the activation of backup communication interfaces for data transfer in any environmental condition.

\section{C.Spectroscopic Mobile and Networkable Station}

Spectroscopic mobile station is a fast and easy deployable gamma and neutron detection system with the capability to be networked wirelessly with other systems.

The station allows response personnel to quickly and safely address radiation threats. It can be positioned in sensitive locations to monitor the situation in real time and in parallel, by both infield personnel and operators at remote command centre.

It provides remote monitoring and turnkey data reach back telemetry capabilities, thereby greatly reducing exposure risk to personnel in compromised environments and allows a discrete monitoring of sensitive locations and events.

\section{D.Portable and Discrete Radionuclide Identifier}

The portable and discrete radionuclide identifier represented in Fig. 6 is a portable detection unit which allows the detection and identification of radiation sources in crowded or sensitive areas or locations forbidden to vehicles.

It is designed to perform undercover measurements in strategic areas like airports or railway stations where the probability for a terroristic attack is higher. It can detect and identify orphan source or radiological dispersal device classifying them in NORM, medical or industrial.

The data are displayed on a visualization device (smartphone or tablet) that allows to remain anonymous in the crowd. In case 
of alarm an acoustic signal, a short vibration or a message can be configured as warning notification.

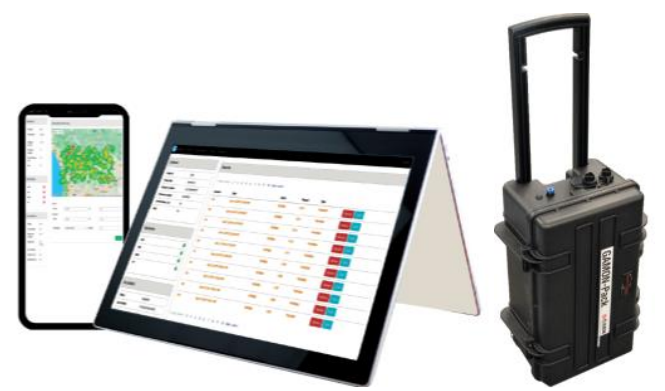

Fig. 6: Picture of the portable and discrete radionuclide identifier

\section{E. Vehicle Mountable Gamma Spectrometric System}

The vehicle mountable gamma spectrometric system is embedded in a rugged and IP66 container designed to resist to external stresses (e.g. shocks and vibrations) and easy to mount and fix on the vehicle chosen for the inspection (boat, helicopter or car), with rechargeable batteries for extended operation.

It is designed to make radiometric measurement and identification of gamma emitters on wide areas providing a map of the radioactivity detected. The real time identification algorithm can recognize artificial gamma emitters beyond the natural background and mark the hot spot over the trajectory travelled by the moving vehicle. This feature allows the user to make a map of the radioactivity of the scanned area.

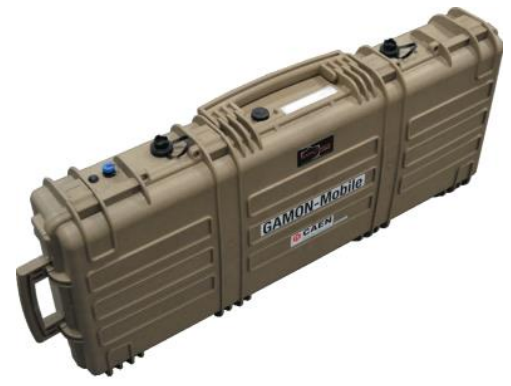

Fig. 7: Picture of the vehicle mountable gamma spectrometric system in its rugged case

\section{F. Compact Radionuclides Identification Unit}

The compact radionuclides identification unit represented in Fig. 8 is an autonomous system designed for radiological threat search. Weight and dimensions allow an easy installation on an UAV or UGV to conduct measurements over large areas to improve the operator safety or for access difficulties.

The system is composed by spectrometric and dosimetry probes for gamma detection integrated in a rugged housing for outdoor environments. It communicates wireless directly to the ground control station where a GIS map visualizes trajectory and measurements without affecting the unmanned vehicle connections and performances.

The system is designed to maximize portability, with a weight lower than $3 \mathrm{~kg}$ that may be further optimized reducing the volume of the installed detector.

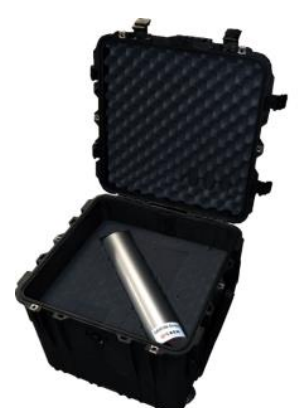

Fig. 8: Picture of the compact radionuclide identification unit in its rugged transportable case

\section{G.Portal Monitor Family}

The radiation monitor systems can be setup to operated portal monitor family for infield real time radiation control of elements passing through the portal for security controls in sensitive access points.

The family is made by two main types of portals: the Pedestrian Portal Monitors (PPM) and the Radiation Portal Monitors (RPM) for the monitoring of vehicles in transit. The systems detect both gamma and neutron emitters thanks to the innovative $\mathrm{NaIL}^{\mathrm{TM}}$ detector which gives the possibility to detect and identify Special Nuclear Material with unprecedented sensitivity, while performing high efficiency gamma spectrometry.

The systems take advantage of a real-time data processing combined to automated background subtraction algorithms to alarm in presence of both artificial nuclides and SNM in a short time window.

\section{H. Underwater System for Radionuclides Identification}

The underwater system for radionuclide identification, represented in Fig. 9, is specifically designed for submerged radiometric measurement and radiological alerts. The system can be used as a quick response measurement device, or it can be installed as a permanent monitoring device for sensitive underwater locations or for oil\&gas applications.

The submersible hermetic case and its weight allow an easy gamma spectroscopic measurement in real time from small boat or watercraft.

The system was designed to offer the best combination of portability, low power consumption and performance.

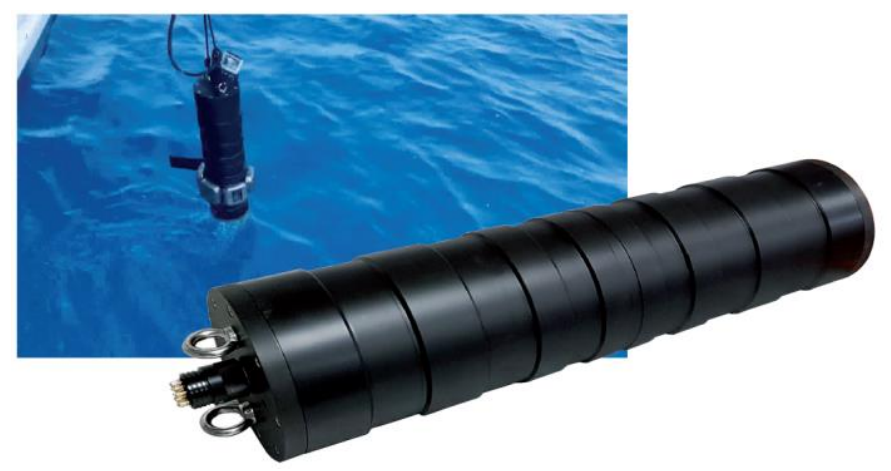

Fig. 9: Picture of the underwater system for radionuclides identification and an on-field use of the system for monitoring the see contamination 


\section{CONCLUSION}

A platform of intelligent radiation monitoring systems with a common web based software gives the possibility to arrange the network of radiation sensors in a modular way. This allows the emergency response teams to choose which type of system shall be deployed and to relocate the monitoring systems depending on the environment, the type of infrastructure or public building to be monitored, the logistic or the geometry of transported material. The real time processing and the connectivity of the systems enhance prompt intervention in case of alarms and allow the decision makers to quickly organize the actions on a contaminated area limiting the operator exposure.

\section{REFERENCES}

[1] YANG K., IEEE Transactions on Nuclear Science PP(99): 1-1 · June 2017

[2] Gamma stream, Digital MCA Tube Base for Gamma-Ray Spectroscopy at http://www.caen.it/csite 